\title{
COMMITMENT DURING NEMATOCYTE DIFFERENTIATION IN HYDRA
}

\author{
TOSHITAF A FUJISA WA AND CHARLES N. DAVID \\ Department of Molezalar Biology, Albert Einstein College of Medicine, Bronx, \\ New York $1046 \mathrm{r}$, U.S.A.
}

\begin{abstract}
SUMMARY
Nematocytes in Hydra differentiate from interstitial stem cells. Desmonemes differentiate mainly in the distal half of the body column while stenoteles differentiate predominantly in the proximal half. This difference was used to determine the timing of nematocyte-type commitment in the differentiation pathway. Cells were transferred from distal or proximal regions to all positions in the body column to test when the proportion of stenotele and desmoneme differentiation changed to reflect the new environment. In the first experiment, the distal region of the body column was isolated and permitted to regenerate a whole $H y d r a$. In the second experiment, dissociated cells from distal or proximal regions were transplanted into regenerating aggregates of $H y d r a$ tissue. Both experiments effectively transferred cells from distal or proximal positions to positions throughout the body column. By comparing the kinetics of stenotele and desmoneme differentiation with the time required for distal or proximal cells to differentiate stenoteles and desmonemes in accord with their new environment, it was possible to conclude that stenotele and desmoneme commitment occurs during the terminal cell cycle prior to nematocyte differentiation and not at the stem cell. Additional experiments indicated that the number of rounds of cell division preceding differentiation is fixed at the time stem cells enter the nematocyte pathway.
\end{abstract}

\section{INTRODUCTION}

Nematocytes in Hydra differentiate from interstitial stem cells (Lehn, 195I; Slautterback \& Fawcett, I959). Four types of nematocytes can be distinguished based on the distinct morphology of the nematocyte capsule: stenotele, desmoneme, holotrichous isorhiza and atrichous isorhiza (Weill, I934). About $90 \%$ of all nematocytes are stenoteles and desmonemes. Stem cells entering the nematocyte pathway undergo 2 to 5 rounds of synchronous cell division (Lehn, I95 I; Rich \& Tardent, I966). Since cytokinesis is incomplete, daughter nematoblasts remain connected to each other by cytoplasmic bridges to form nests of $4,8,16$ or 32 cells (Slautterback \& Fawcett, 1959). Following a terminal cell division, each cell differentiates a nematocyte capsule; all cells in a nest differentiate the same type of capsule (Lehn, I95 I; Rich \& Tardent, I 966). Stenotele nematocytes are produced primarily from nests of 8 and I 6 cells, desmonemes from nests of 16 cells and isorhizas from nests of 8 and 16 cells (David \& Challoner, 1974). Nests of 4 and 32 cells are rare.

Address for correspondence: Dr Charles N. David, Department of Molecular Biology, Albert Einstein College of Medicine, 1300 Morris Park Avenue, Bronx, New York 1046r, U.S.A. 
Nests of 4 nematoblasts are the first cells unique to the nematocyte pathway and, therefore, some kind of 'nematocyte' commitment must precede their formation. However, it is not known when nematoblasts become committed to form a particular nematocyte type. There are 2 basic possibilities:

(I) Nematocyte types are committed at the same time as stem cells are committed to the nematocyte pathway.

(2) Nematocyte types are committed at the later stage in the differentiation pathway, e.g. at the terminal cell division before capsule differentiation.

In order to determine the timing of nematocyte-type commitment in the differentiation pathway we have taken advantage of the fact that the type of nematocyte differention varies along the body column. Desmonemes differentiate mainly in the distal half of the body column while stenoteles differentiate predominantly in the proximal half (Bode \& Smith, 1977). We have used techniques which in effect move cells from one position to another along the body column to determine when in the nematocyte pathway stenotele and desmoneme commitment occurs. In the first experiment we isolated the distal region of the body column and permitted it to regenerate a whole Hydra. Cells in the isolate are effectively transplanted to all positions in the body column by this procedure. In the second experiment, cells from the distal or proximal body column were transplanted into regenerating aggregates of Hydra cells. Such aggregates regenerate normal $H y d r a$ tissue and, thus, cells from distal or proximal regions are effectively transplanted to all positions in the body column. By comparing the time required for the pattern of stenotele and desmoneme differentiation to reflect the new environment with the kinetics of nematocyte differentiation we have shown that stenotele and desmoneme commitment occurs during the terminal cell cycle prior to capsule differentiation.

In the course of these experiments we have also investigated the fate of nests of nematoblasts and nematocytes following dissociation to single cells. The results indicate that cells split from nests of differentiating nematocytes continue differentiation as single cells. Cells split from nests of proliferating nematoblasts continue proliferation and ultimately differentiate nematocytes. The distribution of nest sizes derived from such 'split' nests suggests that the number of cell divisions prior to differentiation is programmed in each cell at the time of initial commitment to the nematocyte pathway.

MATERIALS AND METHODS

Animals

Hydra attenuata were cultured at $19{ }^{\circ} \mathrm{C}$ in the medium containing $\mathrm{I} \mathrm{mM} \mathrm{CaCl}_{2}$, I $\mathrm{mm}$ $\mathrm{NaHCO}_{3}$, and 0.1 mM EDTA. Animals were fed daily with freshly hatched Artemias alinas nauplii and washed $6 \mathrm{~h}$ later.

Animals that had protrusions of their first buds were used for all experiments. The distal or proximal I/5 of the body column was used in all experiments. Regenerating distal pieces were fed daily. 


\section{Cell type analysis}

Cell types were analysed by maceration (David, 1973). Stem cells occur in macerations as large single interstitial cells and in nests of 2 cells. We refer to them as is +28 . We refer to nests of 4 and 8 proliferating cells ( 48 and 88 ) as 'nematoblasts' because they are proliferating cells committed to nematocyte differentiation. We refer to nests of postmitotic cells in which nematocyte capsules are differentiating as 'differentiating nematocytes'. These terms are appropriate given the function and fate of these cell types. It is important to note, however, that in earlier Hydra work nests of nematoblasts were commonly referred to as interstitial cells because of their undifferentiated morphology and nests of differentiating nematocytes were referred to as nematoblasts.

\section{Labelling of cells and autoradiography}

Hydra were labelled with [ $\left.{ }^{3} \mathrm{H}\right]$ thymidine $(100 \mu \mathrm{Ci} / \mathrm{ml})$ by injecting ${ }^{\circ} \mathrm{I} \mu \mathrm{l}$ of the isotope directly into the gastric cavity (David \& Campbell, 1972). Labelled tissue was macerated, and labelled nematocytes were scored by autoradiography using Kodak ARıo stripping film.

\section{Nitrogen mustard (NM) treatment}

Hydra (2000 to 3000 ) were treated with $0.1 \%$ nitrogen mustard for 10 min (Diehl \& Burnett, I 964) and washed thoroughly. Animals were fed once on the day after NM treatment and then starved for 5 to 7 days before they were used for reaggregation experiments.

\section{Culture of interstitial cells and differentiating nematocytes in NM aggregates}

Interstitial cells and differentiating nematocytes were cultured in aggregates of NM-treated Hydra tissue (David \& Murphy, 1977). NM-treated tissue and untreated tissue (plus NMtreated carrier tissue) were each dissociated to single cells in cell culture medium (Gierer $e t$ al. 1972). Appropriate aliquots of untreated cells were mixed with NM-treated cells and aggregates were prepared. Aggregates were incubated as described previously (David \& Murphy, I 977).

\section{Quantitation of differentiating nematocyte nests}

Nests of differentiating nematocytes in regenerating pieces and in aggregates were stained with the thiolacetic acid/lead nitrate reagent (David \& Challoner, 1974). The reagent specifically stains nematocyte capsules for a short period near the end of capsule differentiation. Nests are intact at this stage and thus nest size (number of nematocytes per nest) and nematocyte type can be scored. Since no other Hydra tissue stains with the reagent, stained nests can be scored in whole mounts.

We estimate the duration of the stainable phase to be about $10 \mathrm{~h}$ since the number of desmonemes per animal in macerations is about equal to the number of desmonemes stained by the reagent and the desmoneme population scored in macerations has a turnover time of $10 \mathrm{~h}$ (David \& Gierer, 1974). A similar calculation indicates that stenoteles also are stainable for about io h.

\section{RESULTS}

\section{Differentiation kinetics of nematocytes in regenerating pieces}

The experiments described in this paper use regeneration of the distal body column to test the time of stenotele and desmoneme commitment. To determine the differentiation kinetics of stenoteles and desmonemes in such tissue we pulse-labelled $\mathrm{Hydra}$ with $\left[{ }^{3} \mathrm{H}\right]$ thymidine and $\mathrm{I} h$ later isolated the distal $\mathrm{I} / 5$ of the body column and allowed it to regenerate. Regenerating animals were fed daily. Samples were macerated daily and scored for labelled stenoteles and desmonemes by autoradiography. Labelled 

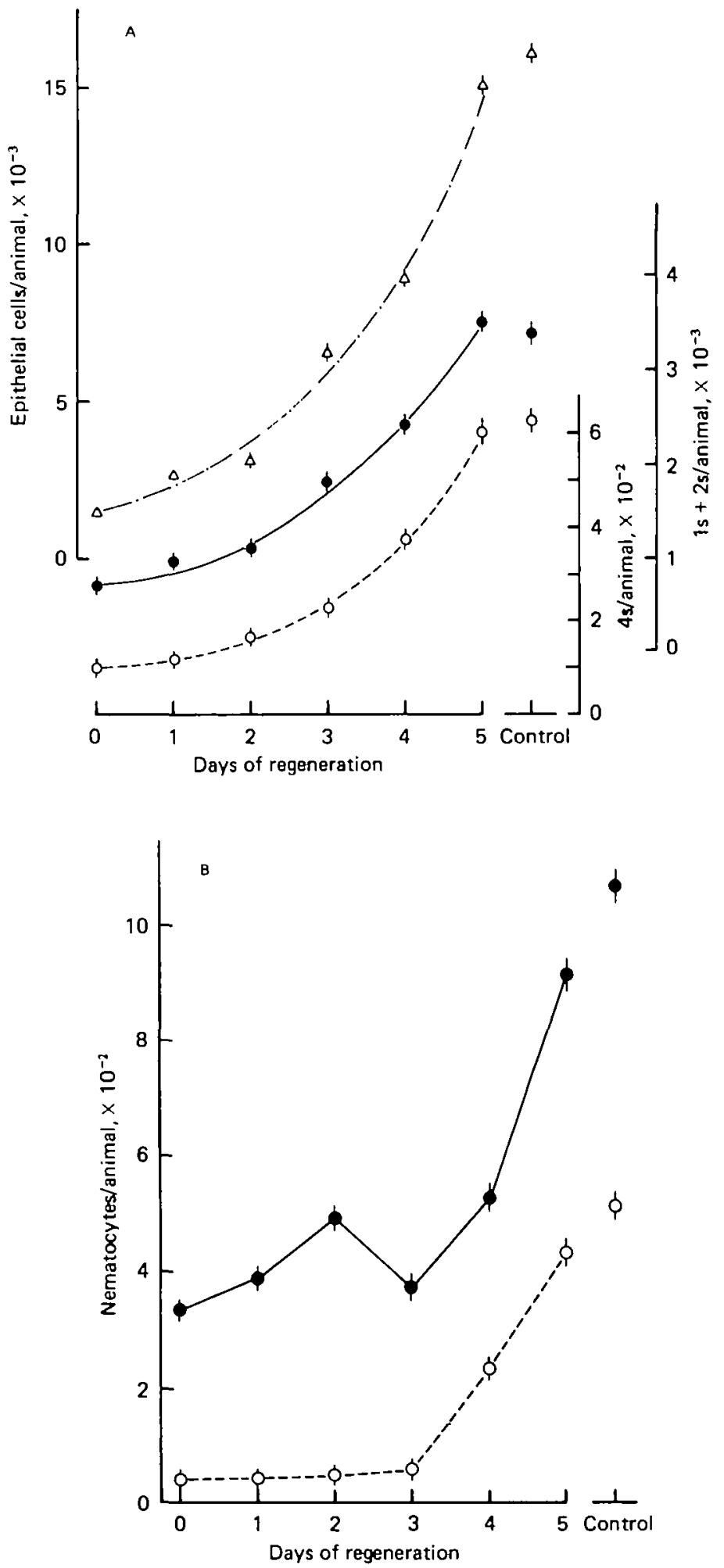

Fig. IA and B. For legend see opposite. 
desmonemes began to appear by $50 \mathrm{~h}$ and labelled stenoteles by $72-84 \mathrm{~h}$. These results are in close agreement with previous observations in intact Hydra (David \& Gierer, 1974) and indicate that the differentiation kinetics of desmonemes and stenoteles are not notably affected by regeneration.

Appearance of stenotele and desmoneme nests during regeneration of distal gastric regions

The distal I $/ 5$ of the body column was excised and allowed to regenerate a normal animal. Cells from the distal region of an animal are effectively transplanted to all positions in the body column by this procedure. As a result the proportions of stenoteles and desmonemes change from those characteristic of the distal region to those characteristic of the whole animal. To score the changes in cell populations during

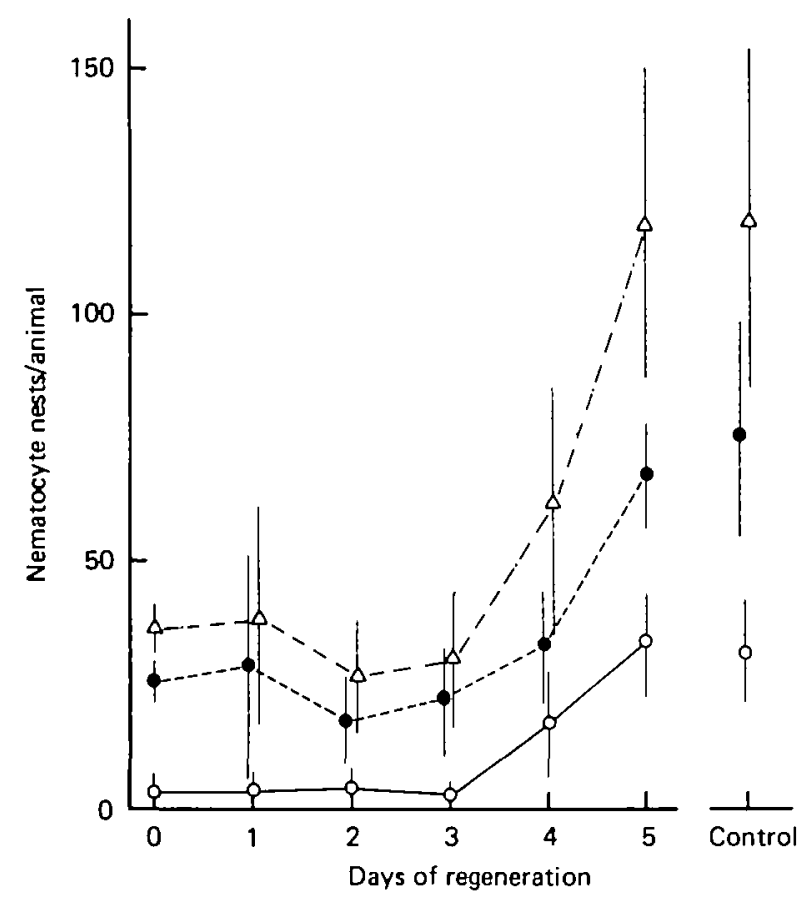

Fig. 2. Changes in the number of nematocyte nests during regeneration of isolated pieces from the distal body column. Differentiating nematocytes were stained with the thiolacetic acid/lead nitrate reagent and scored in whole mounts. Symbols on the right represent the average number of nests in control budding animals. Ten pieces were scored at each point; the error bars indicate the standard deviation. Desmoneme nests, $\bigcirc$ stenotele nests, $O$; total nests, $\triangle$.

Fig. 1. Changes in cell number during regeneration of isolated pieces from the distal body column. Ten pieces were macerated at each time point and the number of each cell type was counted. The experimental error was calculated using the Poisson distribution. Symbols on the right represent the average number of cells in control budding $H y d r a$. A, Epithelial cells, $\triangle$; stem cells (Is $+2 s$ ), 0 ; nematoblasts (4s), $O$. B, Stenoteles, $\mathrm{O}$; desmonemes, 
regeneration, samples were macerated daily and the numbers of epithelial cells, stem cells (Is $+2 s$ ), early nematocyte precursors $(4 s)$ and differentiating stenoteles and desmonemes were counted. The results are shown in Fig. I. The numbers of epithelial cells, Is $+2 \mathrm{~s}$ and $4 \mathrm{~s}$ increased exponentially with a doubling time of about 2 days. By day 5 the regenerating pieces have achieved the size of normal budding Hydra. By

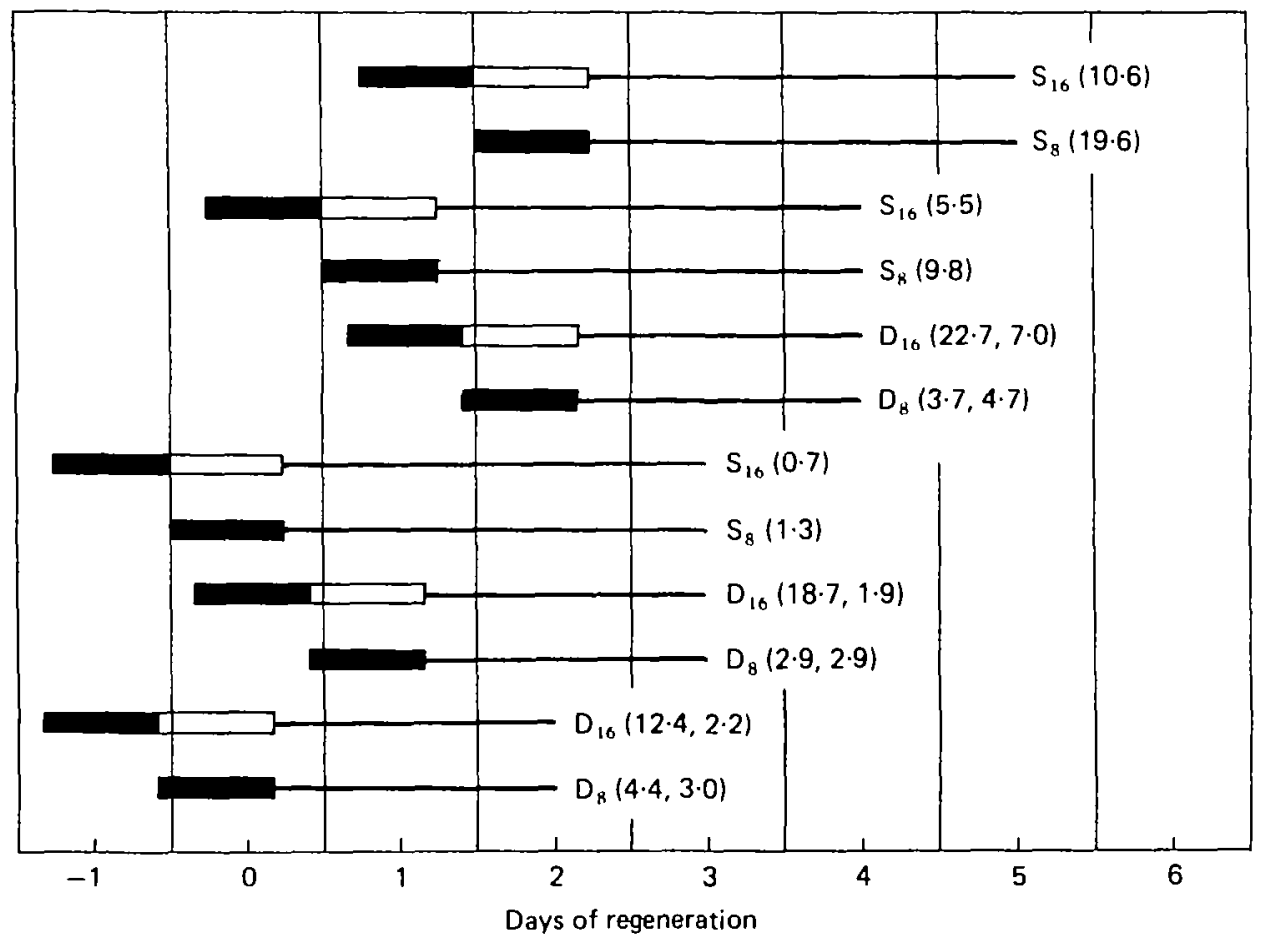

Fig. 3. Schematic representation of the differentiation pathways for nematocyte nests observed in the experiment shown in Fig. 2. Differentiation pathways were constructed using the following parameters: the cell cycle time of 48 and 88 is $18 \mathrm{~h}(12 \mathrm{~h} S$; $6 \mathrm{~h} G_{2}$; Campbell \& David, 1974). The time required for capsule differentiation from the end of the last $S$-phase is $50 \mathrm{~h}$ for desmonemes and isorhizas and $72 \mathrm{~h}$ for stenoteles (David \& Gierer, 1974). To construct the pathway for stenotele nests of $16\left(S_{16}\right)$ on day 4, 4s (filled bars) and $8 \mathrm{~s}$ (open bars) are required on days $O$ and $\mathrm{I}$ followed by 72 -h period of capsule differentiation as shown. The number in parenthesis beside each pathway indicates the number of nests per animal in the pathway (Fig. 2). In the case of desmonemes (D) the first number indicates desmonemes, the second indicates isorhizas which have very similar kinetics. Filled bar, cell cycle time of $4 \mathrm{~s}$. Open bar, cell cycle time of $8 \mathrm{~s}$. Horizontal line, time required for capsule differentiation.

comparison, the numbers of stenoteles and desmonemes did not increase in a simple exponential fashion during this period of regeneration. The number of stenoteles remained almost unchanged for 3 days and then increased rapidly between days 3 and 5 to the normal levels in budding Hydra. By comparison, the number of desmonemes decreased between days 2 and 3 and then increased to normal levels on day 5 .

Since nematocytes differentiate in nests, these changes in the numbers of desmonemes and stenoteles could be due to changes in the number of nests or to changes in 
the average size of nests. In order to distinguish between these possibilities, we have measured the nest size distribution of differentiating stenoteles and desmonemes in regenerating distal pieces using the thiolacetic acid/lead nitrate staining procedure. The results of such an experiment in Fig. 2 show that the total number of stenotele and desmoneme nests changed in parallel with the changes in the number of cells (Fig. IB). The number of stenotele nests remained constant for 3 days and then increased on days 4 and 5 to the normal level in budding Hydra. Desmoneme nests decreased in number between days $I$ and 3 and then increased to normal levels by day 5. Although desmoneme nests showed a decrease on day 2 in the experiment in Fig. 2, this was not commonly observed. Most of our results are similar to the experiment in Fig. I in which the number of desmonemes slightly increases on day 2 and decreases on day 3 .

Figure 3 shows schematically the differentiation pathways for nests of stenotele and desmoneme nematocytes scored in Fig. 2. Nests of 4 and 8 proliferating nematoblasts ( $4 \mathrm{~s}$ and $8 \mathrm{~s}$ ) are indicated by filled and open bars, respectively. By comparing changes observed in Fig. 2 with the differentiation pathways (Fig. 3), it is possible to determine which precursor stage coincides with the onset of regeneration and thus might be the cell responding to changes in positional signals. For example, the increase in stenoteles on day 4 is derived from nematoblast nests whose terminal cell cycle occurred between 12 and $30 \mathrm{~h}$ afrer the start of regeneration. By comparison, stenotele precursors that completed the final cell cycle prior to regeneration or coincident with the start of regeneration did not show an increase. Assuming that positional signals affecting nematocyte commitment change within a few hours after the start of regeneration (Venugopal \& David, I980), it appears that precursors in the final cell cycle before capsule differentiation can sense the new environmental signals and become committed to stenotele differentiation.

The decrease in desmoneme nests (Fig. 2) also suggests that commitment occurs during the terminal cell cycle prior to differentiation or possibly even a few hours later. The decrease in desmoneme nests on day 3 is due to precursors whose final cell cycle occurred between $\mathrm{I} 2$ and $30 \mathrm{~h}$ after the start of regeneration. The decrease in desmoneme nests observed on day 2 is derived from a precursor population which completed its final cell cycle about 4 hours after regeneration. These results suggest that cells at postmitotic stages in the nematocyte pathway may still undergo commitment. However, as noted above this day 2 decrease was not consistently observed (see Fig. I), and thus we favour the conclusion that precursors must spend a significant portion of the terminal cell cycle in the regenerating environment to reflect the commitment pattern of the whole animal. The results of both stenotele and desmoneme differentiation in regenerating distal pieces are thus in good agreement and suggest that commitment of nematocyte types occurs during or very shortly after the terminal cell cycle prior to capsule differentiation.

\section{Fate of nematocyte precursors following dissociation and reaggregation}

An alternative method to test the commitment of stenotele and desmoneme precursors is to transfer cells from distal or proximal regions of the body column into 
regenerating aggregates of $H y d r a$ tissue (Gierer et al. 1972). Such aggregates regenerate with normal proportions of head, gastric and foot tissue. Cells incorporated into such aggregates are thus transplanted effectively to all positions in the body column. By testing when the proportions of stenoteles and desmonemes change from that of distal or proximal tissue to that of the aggregate (total Hydra) environment we can determine which precursors undergo commitment in the aggregate environment.

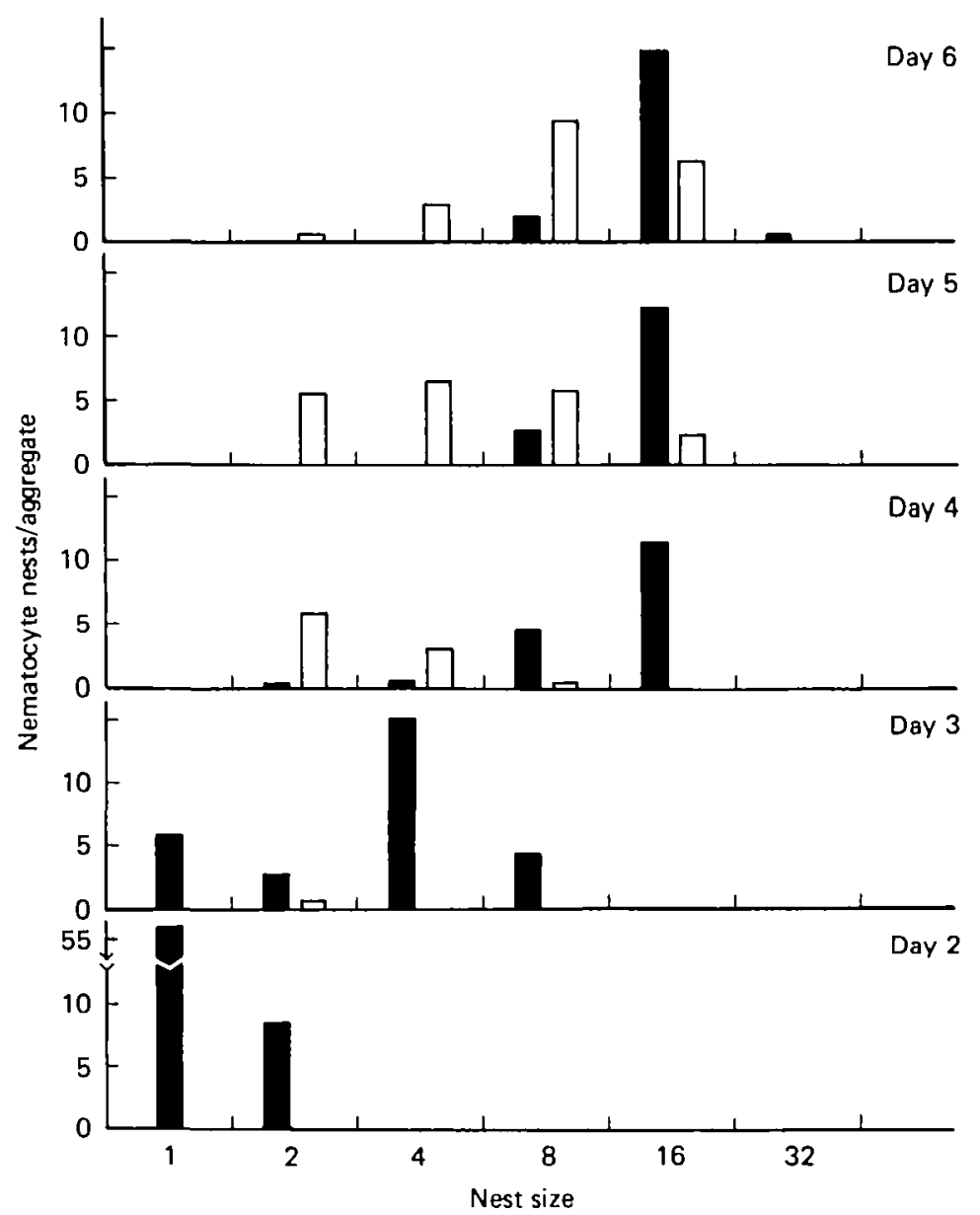

Fig. 4. Nest size distribution of differentiating stenoteles and desmonemes following dissociation and reaggregation. Nematocyte nests were stained with the thiolacetic acid/lead nitrate reagent and scored in whole mounts. The total number of stenotele and desmoneme nests were scored in 8-ro aggregates on each day. Each column indicates the absolute number of nests of that size per aggregate. Filled columns, desmoneme nests. Open columns, stenotele nests.

In a preliminary experiment we investigated the fate of nests of nematoblasts and differentiating nematocytes following dissociation and reaggregation. Cells dissociated from normal $H y d r a$ were mixed with an excess of cells dissociated from NM-treatment Hydra and aggregates were formed. Groups of aggregates were stained daily with the 
thiolacetic acid/lead nitrate reagent and scored stenotele and desmoneme nematocytes. Since NM-treatment eliminates interstitial cells, nematoblasts and differentiating nematocytes from Hydra tissue (Diehl \& Burnett, 1964), the only nematocytes scored in such aggregates are derived from the added normal tissue.

Aggregates prepared from dissociated cells showed a striking and unusual distribution of nematocytes nest sizes (Fig. 4). In particular single desmonemes and stenoteles and small nests of 2 and 4 cells are the only nest sizes observed during the first several days of aggregate regeneration. Such nest sizes are never observed in normal animals. Fig. 4 shows the size distribution of nests of stenotele and desmoneme nematocytes from 2 to 6 days after aggregate formation. The peaks of the nest size distribution shift to larger sizes with increasing time. On day 2 desmonemes occur primarily as single cells. On succeeding days nests of $2,4,8$ and 16 were observed. Thereafter the nest size distribution was similar to that in normal $H y d r a$, where nests of I 6 predominate. Stenoteles showed a similar shift from nests of 2 on day 3 to nests of 8 and 16 on day 6 at which time the distribution was similar to that in normal animals. (Single stenoteles, unlike all other nematocytes, occurred both in the ectoderm and endoderm of aggregates. Those in the ectoderm appeared to be normal differentiating stenoteles, while those in the endoderm appeared to be undergoing degradation. Since these 2 populations could not be distinguished from each other with certainty in all cases, we have not scored single stenoteles.) The shift in stenotele nest size occurred about I day later than the shift in desmoneme nest size in accord with the longer differentiation time of stenotele capsules.

The simplest explanation for the unusual nest sizes and the shift from small nests to large nests during aggregate regeneration is that they represent the continued proliferation and differentiation of nematoblast nests split to single cells by the dissociation procedure. Cells dissociated from a nest of 16 differentiating desmonemes continue differentiation as single cells in aggregates producing single desmonemes. Cells split from a nest of 8 proliferating nematoblasts complete the cell cycle in the aggregate, divide to produce a nest of 2 cells which then differentiate nematocyte capsules. Similarly cells derived from nests of 4 proliferating nematoblasts complete 2 cell divisions in aggregates to yield nests of 4 differentiating nematocytes. The timing of the appearance of the various nest sizes of desmonemes and stenoteles agrees well with this hypothesis. The observation that cells split from proliferating nests at different stages gives rise to unusually small nests suggests that cells in nests are programmed for a limited number of cell divisions before differentiation (see Discussion).

Distribution of nematocyte nests in NM aggregates inoculated with cells from distal and proximal regions of body column

The behaviour of split nests in aggregates provides a convenient way to test the hypothesis that stenotele and desmoneme commitment occurs in the terminal cell cycle. Single stenoteles and desmonemes in aggregates are derived from cells whose terminal mitosis occurred prior to dissociation; nests of 2 and 4 are derived from cells whose terminal mitosis occurred in the aggregates. For these cells at least a portion of the terminal cell cycle occurred in the aggregate environment. By comparing the nest 


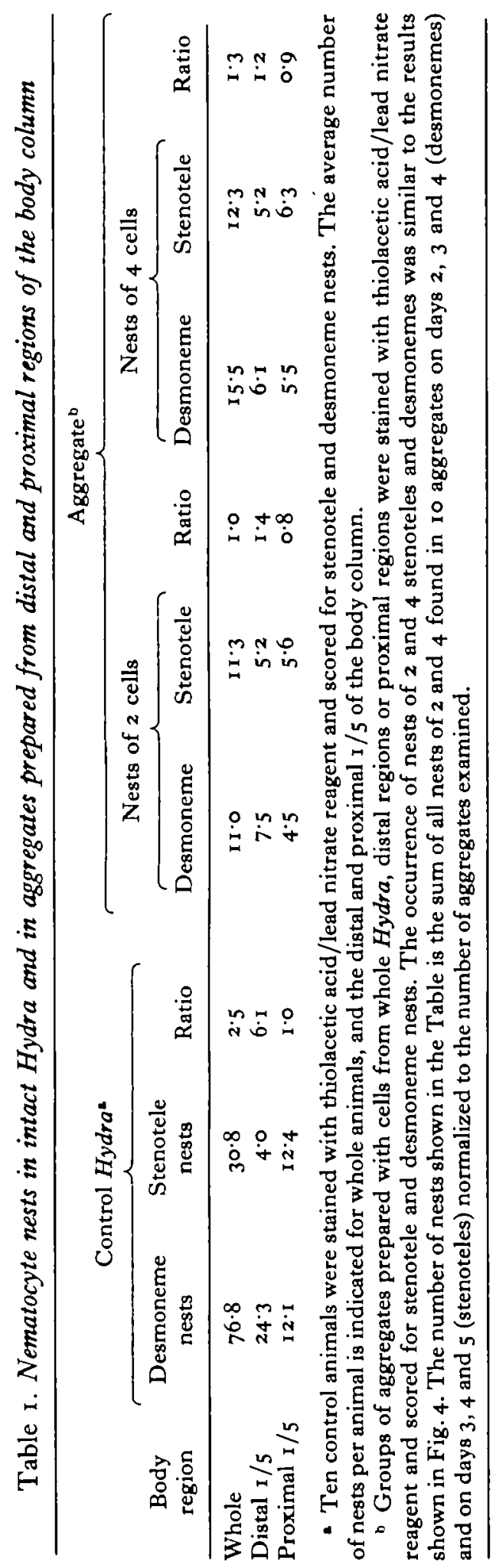


size distribution of differentiating nematocytes in aggregates prepared with cells derived from distal and proximal regions we can determine at what stage of the pathway stenotele and desmoneme commitment occurred. If commitment occurs in the terminal cell cycle, then the proportion of stenoteles and desmonemes in nests of 2 and 4 should be identical in aggregates prepared with distal and proximal cclls since these nests underwent a terminal cell cycle in the aggregate environment. Alternatively, if stenotele and desmoneme commitment occurs prior to the terminal cell cycle, the proportions of stenoteles and desmonemes in nests of 2 and 4 will reflect the proportions characteristic of the distal or proximal tissue added to the aggregates.

The results of such an experiment are shown in Table I. In nests of 2 and 4 nematocytes the ratio of desmonemes to stenoteles is essentially identical in aggregates prepared with cells derived from distal or proximal region or from total Hydra. By comparison the ratio of desmoneme to stenotele nests in normal Hydra differs markedly between distal and proximal regions. The results are thus consistent with the conclusion reached above that stenotele and desmoneme commitment occurs during or after the terminal cell cycle.

The ratio of desmoneme to stenotele nests found in aggregates was consistently lower than that observed in normal animals (Table $\mathrm{r}$ ). This could be due to differences in the plating efficiency of split nests in aggregates or to some difference in the environment of aggregates compared to normal Hydra. However, since the results above indicate that cells giving rise to nests of 2 in aggregates are uncommitted and presumably identical when they enter the aggregate, it is not possible to ascribe to them a difference in plating efficiency. In addition, the apparent plating efficiency of stenotele nests of 2 in the present experiments is about $50 \%$ which is so high as to be unlikely. (The plating efficiency of stem cells in aggregates is about $15 \%$; David \& Murphy, 1977.) Thus we favour the idea that aggregate environment is somehow different from the environment in normal Hydra and that it favours stenotele commitment.

\section{DISCUSSION}

\section{The timing of stenotele and desmoneme commitment}

The proportion of stenotele and desmoneme differentiation in $\mathrm{Hydra}$ depends on position in the body column. Distal positions favour desmoneme differentiation; proximal positions favour stenotele differentiation. We have used this difference to determine the time of stenotele and desmoneme commitment in relation to the nematocyte differentiation pathway. Our approach was to transfer cells from distal or proximal regions to all positions in the body column and to test when the proportions of stenotele and desmoneme differentiation changed to reflect the new environment. The results of 2 different experiments were in good agreement and suggested that proliferating nests of nematoblasts become committed for stenotele or desmoneme differentiation at or near the final cell cycle prior to capsule differentiation. Thus stenotele and desmoneme commitment clearly occurs at a later stage than commitment of stem cells to enter the 'nematocyte' pathway. 
The present experiments do not permit us to localize the commitment event more precisely. This is partly due to technical difficulties with the experiments and partly due to insufficient knowledge about the nature of the positional signals which control stenotele and desmoneme commitment. In the case of regenerating distal pieces, the results clearly indicate that commitment did not occur before the terminal cell cycle. However, the results left open the possibility that commitment might extend past the terminal cell cycle into the early stages of differentiation. Similarly the aggregation experiment clearly indicated that cells whose terminal cell cycle occurred in the aggregates were committed in aggregates. However, our inability to score single stenoteles in aggregates prevented us from deciding if commitment can occur at a somewhat later stage. Although neither experiment eliminates the possibility of commitment during early stages of differentiation after the terminal cell cycle, this possibility appears unlikely for 2 reasons. (I) We have shown that treatment of regenerating distal pieces with hydroxyurea inhibits the increase in stenotele differentiation in Fig. I B (Fujisawa \& David, unpublished results). Since hydroxyurea blocks cells in $S$-phase (Bode, Flick \& Smith, 1976), this result suggests that precursors in the terminal cell cycle, not postmitotic cells, are the cell population responding to the new environment in regenerating distal pieces. (2) The differentiation pathways of stenoteles and desmonemes appear to be divergent cytologically. Thus a change from one to the other seems more likely before rather than after the start of overt cytodifferentiation (which begins after the last mitosis). Hence we favour the idea that stenotele and desmoneme commitment occurs during the terminal cell cycle.

The results of 2 earlier experiments designed to test nematocyte commitment also suggest that commitment occurs during the terminal cell cycle. Lehn (I95I) and Tardent, Rich \& Schneider (197I) used the distribution of large and small stenoteles along the body column and a regeneration experiment similar to ours to determine the time of stenotele commitment. Large stenoteles are localized in the distal body column, small stenoteles in the proximal body column. When Hydra were cut in half and both pieces allowed to regenerate, small stenoteles appeared in the distal half and large stenoteles in the proximal half in about 5 days. When these results are compared with the differentiation times of stenotele capsules (David \& Gierer, 1974), it appears likely that commitment of large and small stenoteles occurs in nests just prior to capsule differentiation in agreement with the conclusion of the present experiments.

In an alternative approach, Zumstein \& Tardent (197I) and Zumstein (1973) investigated the differentiation of stenoteles following selective depletion of stenoteles in the tentacles. Their results indicated that stenotele populations recovered in size between 5 and 7 days after treatment. The recovery closely paralleled the differentiation of labelled stenoteles from precursors labelled with $\left[{ }^{3} \mathrm{H}\right]$ thymidine at the time of stenotele depletion. Since nests labelled during the terminal cell cycle are the first to differentiate labelled stenoteles, these results also indicate that nematoblast nests, not stem cells, are the cell population committed to stenotele differentiation in response to stenotele depletion in the tentacles. 


\section{Quantitative shift of precursors from desmoneme to stenotele pathway}

The experiments above strongly suggest that stenotele and desmoneme commitment occurs in the terminal cell cycle. This indicates that proliferating nests in the nematocyte pathway are uncommitted. Since the number of nests is fixed, an increase in stenotele differentiation must occur at the expense of a decrease in desmoneme differentiation. Indeed the results in Figs. I and 2 suggest that this may be true: the number of desmoneme nests decreased on days $1-3$ while the number of stenotele nests increased on days $3-5$. The difference in timing is due to the longer differentiation time of stenoteles compared to desmonemes. In the following calculations we have tested this idea quantitatively.

Table 2. Comparisons of observed and calculated numbers of nematoblast nests in regenerating distal pieces

\begin{tabular}{|c|c|c|c|c|}
\hline \multirow{2}{*}{$\begin{array}{l}\text { Days of } \\
\text { regeneration }\end{array}$} & \multicolumn{2}{|c|}{$\begin{array}{l}\text { Observed number of nests } \\
\text { per regenerate }\end{array}$} & \multicolumn{2}{|c|}{$\begin{array}{l}\text { Calculated number of nests required } \\
\text { for nematocyte differentiation }\end{array}$} \\
\hline & 43 & 88 & 48 & $8 s$ \\
\hline 0 & $94 \cdot 8$ & 40.5 & 59.5 & $27 \cdot 7$ \\
\hline I & 1254 & $49 \cdot 7$ & 104.7 & $49 \cdot 3$ \\
\hline 2 & I $94^{\circ} 9$ & $78 \cdot 4$ & $187 \cdot 1$ & $78 \cdot 1$ \\
\hline
\end{tabular}

- Scored in macerations on days 0,1 and 2.

b The number of nematoblast nests (4s and 88 ) required for each differentiation pathway shown in Fig. 3 was calculated as described in the text. For example, the number of 49 on day 0 in the pathway leading to $S_{16}$ on day 4 is:

$5.5 S_{10}$ nests $\times \frac{18 \mathrm{~h} 48 \text { cell cycle }}{10-\mathrm{h} \text { staining period }} \times 1 \cdot 0$ (fraction of 48 cell cycle occurring on day 0$)$.

Similar calculations were done for all 48 and 8 s present on days 0,1 and 2 in each pathway in Fig. 3. The total number of $4 \mathrm{~s}$ and $8 \mathrm{~s}$ on each day was then calculated by summing the contribution from each pathway.

From the results in Fig. 3 it is possible to calculate the number of nests of 4 and 8 nematoblasts ( $4 \mathrm{~s}$ and $8 \mathrm{~s}$ ) entering each differentiation pathway (Table 2). For example, there were an average of 5.5 nests of 16 stenoteles per regenerate on day 4 . Correcting this number for the duration of the staining period ( $10 \mathrm{~h})$, the duration of the $4 \mathrm{~s}$ cell cycle ( $18 \mathrm{~h}$; Campbell \& David, I974), and the fraction of the cell cycle occurring on day $\circ(\mathrm{I} \cdot 0)$, it is possible to estimate the number of 48 on day $\circ$ which contribute to this pathway. Similar calculations were made for all 48 and $8 \mathrm{~s}$ in Fig. 3 .

The total number of $4 \mathrm{~s}$ and $8 \mathrm{~s}$ on each day was then calculated by summing the contribution from each line in Fig. 3. These are the total precursor cells required to support the observed levels of nematocyte differentiation on later days during regeneration. These calculated numbers of $4 \mathrm{~s}$ and $8 \mathrm{~s}$ are in good agreement with actual measurements of 48 and $8 \mathrm{~s}$ made on parallel samples using the maceration procedure (Table 2). This agreement indicates that all $4 \mathrm{~s}$ and $8 \mathrm{~s}$ can be accounted for as precursors 
to nematocyte nests on later days. Thus, as in normal Hydra (David \& Gierer, 1974), proliferating nests appear only to function as precursors to nematocytes.

From the results in Fig. 3 and Table 2 it is possible to calculate the proportions of $4 \mathrm{~s}$ and $8 \mathrm{~s}$ which enter each differentiation pathway on each day. These results are shown in Table 3 along with similar calculations performed on the distal gastric region of non-regenerating Hydra. The calculations indicate that day 0 regenerates are similar to control $H y d r a$ in terms of the proportion of $4 \mathrm{~s}$ and $8 \mathrm{~s}$ differentiating as 8 and I6 nematocytes. There is, however, a significant difference in the proportion of $4 \mathrm{~s}$

Table 3. Changes in the proportion of 45 and $8 s$ undergoing desmoneme or stenotele nematocyte differentiation during regeneration of isolated distal pieces

\begin{tabular}{|c|c|c|c|c|c|c|c|c|}
\hline \multirow{2}{*}{$\begin{array}{l}\text { Nematocyte } \\
\text { pathway }\end{array}$} & \multicolumn{4}{|c|}{$\begin{array}{c}\% \text { of } 48 \text { committed on each day } \\
\text { of regeneration }\end{array}$} & \multicolumn{4}{|c|}{$\begin{array}{c}\% \text { of } 8 \text { s committed on each day } \\
\text { of regeneration }\end{array}$} \\
\hline & Control $^{b}$ & 0 & I & 2 & Control ${ }^{b}$ & 0 & I & 2 \\
\hline $\mathrm{D}_{8}$ & $2 \cdot 4$ & $12 \cdot 3$ & 4.9 & 3.4 & & & & \\
\hline$D_{16}$ & $69 \cdot 2$ & 53.9 & $37 \cdot 2$ & $55 \cdot 2$ & $85 \cdot 0$ & $8 I \cdot 2$ & $66 \cdot 5$ & $59^{\circ} \circ$ \\
\hline $\mathrm{S}_{8}$ & $7 \cdot 1$ & $3 \cdot 7$ & $21 \cdot 0$ & $20 \cdot 0$ & & & & \\
\hline$S_{18}$ & $5 \cdot 3$ & 15.8 & $20 \cdot 4$ & 10.8 & $6 \cdot 5$ & $5 \cdot 1$ & 24.9 & 25.9 \\
\hline$I_{8}, 16$ & 16.0 & 14.3 & 16.5 & $10 \cdot 6$ & $8 \cdot 5$ & 13.7 & $8 \cdot 6$ & $15 \cdot 1$ \\
\hline
\end{tabular}

The proportion (\%) of 48 and $8 \mathrm{~s}$ which enters each differentiation pathway on each day was calculated from the results in Fig. 3 and Table 2. Similar calculations were done on the distal gastric region of intact budding Hydra.

- $\mathrm{D}_{8}$, nests of 8 desmonemes; $\mathrm{D}_{16}$, nests of 16 desmonemes; $\mathrm{S}_{8}$, nests of 8 stenoteles; $S_{18}$, nests of 16 stenoteles; $I_{8}, 18$, nests of 8 and $I 6$ isorhizas.

D Control, distal I / 5 body column of intact budding Hydra.

which go on to differentiate as 16 nematocytes: the proportion of 4 s giving rise to 16 desmonemes is decreased and the proportion giving rise to 16 stenoteles is increased. On day $\mathrm{I}$ of regeneration the most conspicuous change in nematocyte differentiation is the shift of about $20 \%$ of $8 \mathrm{~s}$ from the desmoneme to the stenotele pathway. There is also an increase in $4 s$ giving rise to 8 stenoteles and a somewhat smaller decrease in $4 \mathrm{~s}$ differentiating 8 desmonemes. (This latter change may be an artefact of this experiment since the day o value is unusually high compared to the control in Table 3 as well as compared to other control Hydra we have examined.)

The calculations in Table 3 demonstrate 2 important points. First, the observations support the conclusion that commitment occurs in the terminal cell cycle. Nests which differentiate after I division in regenerates show changes in proportions of stenoteles and desmonemes on day $\mathrm{I}$; nests which differentiate after 2 divisions show changes in proportions on day 0 . Since the latter cells actually undergo their terminal division on day I in regenerates, these observations are consistent with each other. Both support the conclusion that commitment occurs in the terminal cell cycle. Second, by accounting for all nematoblast nests (Table 2 and 3 ), we can exclude the possibility that the changes in stenotele and desmoneme differentiation simply reflect the differential survival of already committed nests. Since we can demonstrate that nematoblast nests 
change from the desmoneme to the stenotele pathway under the influence of regeneration, we can conclude that these nests were uncommitted at the start of regeneration.

\section{'Split' nests and the control of cell division during nematocyte differentiation}

Interstitial stem cells committed to nematocyte differentiation undergo a series of synchronous cell divisions to form nests of 8 and I 6 (occasionally 4 and 32) cells, which then differentiate nematocyte capsules. The observation that nematocyte differentiation occurs from a narrowly distributed set of nest sizes suggests some form of control over the number of cell divisions in the pathway. At least 2 alternatives can be proposed: ( $\mathrm{I}$ ) the number of cell divisions is programmed at the time a stem cell initiates nematocyte differentiation. In this model capsule differentiation would occur after a fixed number of cell divisions. (2) The number of cell divisions depends on some measure of cell number per nest at which point proliferation ceases and differentiation begins. In this model nests need to achieve a critical size in order to differentiate.

These 2 possibilities can be distinguished by following the fate of cells split from proliferating nests. The first model predicts that capsule differentiation would occur in the components of split nests after the programmed number of cell divisions thus giving rise to unusually small nests. The alternative model predicts that the components of split nests would continue proliferation until a new nest of the appropriate size for differentiation had been achieved. The results in Fig. 4 indicate that unusually small nests of differentiating nematocytes are observed following dissociation of proliferating nests to single cells. This strongly suggests that the number of cell divisions preceding differentiation is programmed in cells of proliferating nests. Individual cells appear to count mitoses and differentiate after 3 or 4 cell divisions even in the absence of their normal neighbours in the nest. In this regard proliferating nematoblasts are very similar to other proliferating blast cells. For example, sperm and egg differentiation in many systems is preceded by a fixed number of divisions (Fawcett, 196r; Clermont, 1972; King, 1970). Similarly differentiation of murine erythroleukemia cells is preceded by 4 cell divisions (Gusella et al. 1976) and differentiation of erythrocytes in chick embryos is preceded by 6 cell divisions (Weintraub, Campbell \& Holtzer, 1971).

The authors thank Dr H. K. MacWilliams for critical review of the manuscript. This research was supported by grants from the NIH (GM II3OI) and the NSF (77-25426). C.N.D. is recipient of a Career Development Award (FRA-132) from the American Cancer Society. T.F. is recipient of a fellowship from the Japanese Ministry of Education.

\section{REFERENCES}

BODE, H. R., FLICK, K. M. \& SMITH, G. S. (I 976). Regulation of interstitial cell differentiation in Hydra attenuata. I. Homeostatic control of interstitial cell population size. 9 . Cell Sci. 20, 29-46.

Bode, H. R. \& SмIтh, G. S. (1977). Regulation of interstitial cell differentiation in Hydra attemuata. II. Correlation of the axial position of the interstitial cell with nematocyte differentiation. Wilhelm Roux Arch. EntwMech. Org. 181, 203-213.

Campbell, R. D. \& David, C. N. (1974). Cell cycle kinetics and development of $\mathrm{Hydra}$ attemuata. II. Interstitial cells. F. Cell Sci. 16, 349-358. 
Clermont, Y. (1972). Kinetics of spermatogenesis in mammals: seminiferous epithelium cycle and spermato-gonial renewal. Physiol. Rev. 52, 198-236.

David, C. N. (1973). A quantitative method for maceration of Hydra tissue. Wilheln Roux Arch. EntwMech. Org. 17x, 259-268.

David, C. N. \& CAMPBell, R. D. (1972). Cell cycle kinetics and development of Hydra attenuata. I. Epithelial cells. 7. Cell Sci. Ir, 557-568.

David, C. N. \& Challoner, D. (1974). Distribution of interstitial cells and differentiating nematocytes in nests in Hydra attenuata. Am. Zool. 14, 537-542.

David, C. N. \& Gierer, A. (I974). Cell cycle kinetics and development of Hydra attenuata. III. Nerve and nematocyte differentiation. F. Cell Sci. 16, 359-375.

David, C. N. \& MuRPhy, S. (1977). Characterization of interstitial stem cells in Hydra by cloning. Devl Biol. 58, 372-383.

Diehl, F. \& BurnetT, A. L. ( 1964$)$. The role of interstitial cells in the maintenance of Hydra. I. Specific destruction of interstitial cells in normal, sexual and non-budding animals. 7. exp. Zool. r55, 253-259.

FAwCETt, D. W. (i 96i). Intercellular bridges. Expl Cell Res., Suppl. 8, i 74-i 87.

Gierer, A., Berking, S., Bode, H. R., David, C. N., Flick, K. M., Hansmann, G., Schaller, H. \& TrenkNER, E. (1972). Regeneration of Hydra from reaggregated cells. Nature, New Biol. 239, 98-IOI.

Gusella, J., Geller, R., Clarke, B., Weeks, V. \& Housman, D. (1976). Commitment to erythroid differentiation by Friend erythroleukemia cells: A stochastic analysis. Cell 9, $221-229$.

KING, R. C. (1970). Ovarian Development in Drosophila melanogaster. New York and London: Academic Press.

LEHN, H. (I95I). Teilungsfolgen und determination von I-zellen für die Cnidenbildung bei Hydra. Z. Naturf. 6b, 388-39r.

Rich, R. \& TARDENT, P. (ig66). Untersuchung zur Nematocyten-Differenzierung bei $H y d r a$ attenuata. Rev. suisse Zool. 76, 779-789.

SlautTERBack, D. B. \& FawCETT, D. W. (1959). The development of the cnidoblasts of $H y d r a$. An electron microscope study of cell differentiation. F. biophys. biochen. Cytol. 5, 44I-452.

TARdent, P., Rich, R. \& SchneIder, V. (I97I). The polarity of stenotele differentiation in Hydra attenuata Pall. Devl Biol. 24, 596-608.

Venugopal, G. \& David, C. N. (1 980). Nerve commitment in Hydra. I. Role of morphogenetic signals. Devl Biol. (In Press.)

Weill, R. (1 934). Contribution a l'étude des cnidaires et du leurs nematocystes. Trav. Stn zool. Wimereux I0, 1I, I-70I.

Weintraub, H., Campbell, G. Le M. \& Holtzer, H. (1971). Primitive erythropoiesis in early chick embryogenesis. I. Cell cycle kinetics and the control of cell division. $\mathcal{J}$. Cell Biol. 50, $652-668$.

Zumstein, A. (1973). Regulation der Nematocyten-Produktion bei Hydra attenuata Pall. Wilhelm Roux Arch. EntwMech. Org. 173, 294-3 18.

Zumstein, A. \& Tardent, P. (1971). Beitrag zum Problem der Nematocyten-Produktion bei Hydra attemuata Pall. Rev. suisse Zool. 78, 705-714.

(Received 14 August 1980) 